\title{
Application of Green Catalysts for the Esterification of Benzoic Acid with Different Alcohols
}

\author{
Emine Sert*, Ferhan S. Atalay \\ Ege University, Chemical Engineering Department, Bornova, Izmir, Turkey \\ 232 3111493, fax. 2323887600 \\ *emine.sert@ege.edu.tr \\ *Corresponding Author \\ Received: 13 July 2017 \\ Accepted: 28 November 2017 \\ DOI: $10.18466 /$ cbayarfbe. 370364
}

\begin{abstract}
In this study, three different catalysts were applied as catalysts for esterification of benzoic acid with ethanol, butanol and hexanol. These catalysts are ionic liquid, deep eutectic solvent, ion exchange resin. Amberlyst 15, ion exchange resin, was used as control catalyst to observe the catalytic activity of new generation solvent, deep eutectic solvent. Deep eutectic solvents show the similar properties with ionic liquids and have great attraction due to ease of preparation, low cost and environmentally friendly nature. They have many advantages such as reusability, stability, catalytically activity and removal of the reaction mixture. Deep eutectic solvents (DES) are composed hydrogen bond donor (HBD) and hydrogen bond acceptor (HBA). It has lower freezing point than that of individual constituents. In this study, $p$-toluene sulfonic acid (p-TSA) was used as HBD, benzyl tri-ethyl ammonium chloride (BTEAC) was used as HBA. DES formed by p-TSA and BTEAC was used as dual solventcatalyst for esterification of benzoic acid with different alcohols. DES gave high catalytic activity among three different catalysts, 88.3, 87.8 and $67.5 \%$ conversion of benzoic acid for ethanol, butanol and hexanol, respectively. The effects of time, temperature, alcohol type and catalyst type were investigated in a batch reactor at specified conditions. It was found that DES provided the simple, efficient and environmentally friendly method for the synthesis of benzoic acid ester.
\end{abstract}

Keywords - benzoic acid, catalysis, deep eutectic solvent, esterification, ionic liquid, ion exchange resin

\section{Introduction}

Synthesis of organic esters has a great role in organic synthesis and very large number of products of industrial interest is produced by the reaction between an organic acid and an alcohol. So, high ester use in the synthesis of drugs, fine chemicals, pharmaceuticals, solvents and plasticizers as intermediates makes these substrates one of the most important types of compounds in organic chemistry [1]. The synthesis of carboxylic esters involves the reaction of carboxylic acids with alcohols using suitable acidic catalysts.

Esterification of a carboxylic acid with an alcohol is very slow in the absence of catalyst. The extent of reaction of carboxylic acid is limited by the equilibrium constant, so that the complete conversion cannot be obtained [2]. So, use of suitable catalyst becomes more important. The catalytic activity, stability and reusability are the major concerns for the industrial applications to increase the rate of reaction. The popular catalysts for esterification reactions are homogeneous catalysts such as sulfuric acid, hydrochloric acid and heterogeneous catalysts such as ion exchange resins.

The traditional catalysts for esterification reactions are generally homogeneous catalysts [3-5], but these catalysts have several drawbacks; corrosion, separation from reaction mixture, side reactions and also environmental aspects. Instead of homogeneous catalysts, heterogeneous catalysts such as ion exchange resins [6], zeolites [7], supported heteropolyacids [8] have been used as acidic catalysts to accelerate the esterification reactions. However, many of these solid catalysts suffer from inherent drawbacks such as the need for expensive or harmful materials as reagents and catalysts, the formation of undesired side products, the use of hazardous and toxic solvents, the requirement of high reaction temperatures and prolonged reaction times, synthesis of low yield of products [9]. In addition, one of the most important disadvantages of using solid catalysts in benzoic acid esterification reactions is requirement of organic solvents. 
The use of organic solvent creates waste and purification problems. Nowadays, new catalysts are being developed for synthesizing esters to minimize the pollution caused by traditional methods. Catalyst should have superior properties such as minimum waste disposal in addition to high catalytic activity and thermal stability.

Among the organic esters, benzoic acid esters are used in production of dyes, heat resistant lubricants, herbicides, alkali-resistant polyester coatings, and also as plasticizers for synthetic resins, cellulose ethers and esters, rubbers, and paper, as additives for dyeing fabrics made from manmade fibers, as polymerization catalysts, drugs, active ingredients of plant growth regulators, and cosmetic means [10]. Because of the industrial importance of benzoic acid esters, catalyst plays an important role in the esterification of benzoic acid. To synthesize high active catalytically, reusable, stable and also environmentally friendly catalyst for production of benzoic acid esters, is the main purpose of this study.

Recently, ionic liquids (ILs) have attracted more in various chemical processes due to their many superior properties such as nonvolatile, thermally stable properties, and ease of recyclability [11]. Chai et.al [11] studied the esterification of benzoic acid with different alcohols catalyzed by several ionic liquids. $\left[\mathrm{C}_{3} \mathrm{SO}_{3} \mathrm{Hmim}\right] \mathrm{HSO}_{4}$, $\left[\mathrm{C}_{3} \mathrm{SO}_{3} \mathrm{Hmim}\right] \mathrm{BF}_{4}, \quad\left[\mathrm{C}_{3} \mathrm{SO}_{3} \mathrm{Hmim}\right] \mathrm{PF}_{6}, \quad$ and $\left[\mathrm{C}_{3} \mathrm{SO}_{3} \mathrm{Hmim}\right] \mathrm{CF}_{3} \mathrm{SO}_{3}$ are used as catalysts and catalytic activities were found at the end of 3 hours. Using different ionic liquids high conversions of benzoic acid were achieved. $\left[\mathrm{C}_{3} \mathrm{SO}_{3} \mathrm{Hmim}\right] \mathrm{HSO}_{4}$ showed good catalytic activities in esterification of other alcohols like methanol, butanol, cyclohexanol, benzenemethanol, and lauryl alcohol with good to excellent yields (83-98\%).

Owing to their low vapor pressure and high boiling point, which facilitates their recycling, ILs were qualified as green solvents, but many studies pointed out the hazardous toxicity and poor biodegradability of ILs. Also, their synthesis is far to be environmentally friendly since it generally requires a large amount of salts and solvents in order to completely exchange the anions. These disadvantages combine with the high price, ILs become less attractive for the industrial applications. [12]. Deep eutectic solvents (DES) have recently appeared as a new generation of ionic solvents of low cost and biodegradable. They are a combination of a hydrogen bond acceptor (HBA) and a hydrogen-bond donor (HBD), in which a new compound is formed, usually having much lower melting temperature than the components of the combination. DESs are advantageous because they can be easily prepared in high purity at low cost, and their components are biodegradable and possess low toxicity
[13]. The use of DES is esterification reactions as both catalyst and solvent is limited [14-16]. De Santi [14] et.al studied the esterification of different acids and alcohols catalyzed by deep eutectic solvents. p-Toluenesulfonic acid and different ammonium salts were used as HBA and HBD, respectively. According to synthesized ester and used DES, different conversion values achieved between $40-97 \%$.

In this study, we have tried to synthesize benzoic acid esters in simple, efficient, lower costly, environmentally friendly and recyclable way. (a) Esterification of benzoic acid with ethanol was carried out three different catalysts, DES (composed of p-TSA and BTEAC, Figure 1), ionic liquid (1-Butyl-3-metylimidazolium chloride) and ion exchange resin (Amberlyst 15) for comparison. (b) After deciding the most efficient catalyst, effect of alcohol was investigated. (c) Effect of temperature was obtained for all alcohols and catalysts. There is no any study including the esterification of benzoic acid catalyzed by deep eutectic solvents.
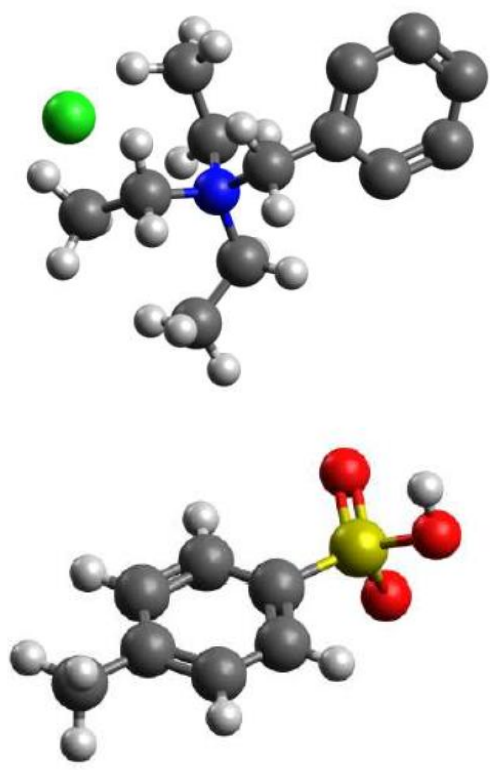

Figure 1. Chemical structure of BTEAC and $p$-TSA (green: $\mathrm{Cl}$, dark grey: $\mathrm{C}$, light grey: $\mathrm{H}$, blue: $\mathrm{N}$, yellow: $\mathrm{S}$, red: o)

\section{Experimental Study \\ 2.1 Materials}

In this study, benzoic acid, ethanol, n-butanol and hexanol were used as reactants. Ionic liquid (1-Butyl-3metylimidazolium chloride), deep eutectic solvent (formed by p-toluene sulfonic acid and benzyl triethyl ammonium chloride) and ion exchange resin (Amberlyst 
15) were used as catalysts.

\section{$\underline{D E S}$}

As stated earlier, deep eutectic solvent was prepared by mixing certain amounts of HBA and HBD. The preparation method was explained in our previous study [15]. The mixtures containing different amounts of HBA and HBD were heated and stayed to cool. The freezing points of each mixture were obtained and the composition of HBA which gives the minimum freezing point was designated as eutectic point. In our previous study, eutectic point of DES was found as 0.3-0.7 (HBA-HBD ratio) and corresponding freezing point was $2^{\circ} \mathrm{C}$. The physical properties give the idea about applicability of DES in any chemical process. Also, $\mathrm{pH}$ is important parameter in addition to physical properties because of the requirement of acidic catalyst. Density, viscosity, refractive index and $\mathrm{pH}$ values were given [16]. Density, viscosity and $\mathrm{pH}$ of synthesized DES were found as $1.1668 \mathrm{~g} / \mathrm{cm}^{3}, 12164 \mathrm{cP}$ and -1 at room temperature.

\section{Ionic Liquid}

In this study, 1-Butyl-3-metylimidazolium chloride is used as a catalyst. Molecular formula is $\mathrm{C}_{8} \mathrm{H}_{15} \mathrm{ClN}_{2}$ and molecular weight is $174.67 \mathrm{~g} / \mathrm{mol}$.

\section{Ion exchange resin}

Amberlyst 15 was used ion exchange resin. Ionic form of resin is hydrogen and resin is macroporous, acidic, styrene divinyl benzene copolymer [17].

\subsection{Experimental Procedure}

The experimental setup consists of reactor, heater equipped with temperature controller, magnetic stirrer and condenser. The heater gives the required energy to increase the reaction temperature to desired value. Temperature controller is used to maintain the reactor temperature at this value. Also magnetic stirrer provides the uniform mixing of reaction mixture; uniform temperature and concentration distribution are assumed in reactor. The coolant circulating through condenser prevents the escaping of part of reaction mixture.

In a typical run, benzoic acid and catalyst (DES or ionic liquid or ion exchange resin) were fed to the reactor with a specified amount. Temperature was set to desired value, and then heated alcohol was added to acid-catalyst mixture. During the experiments, catalyst loading was kept constant at $10 \mathrm{wt} \%$ for all catalysts and also alcoholacid mole ratio was kept constant at 10 . The weight of catalyst was determined on the basis of weight of the reaction mixture. Firstly, experiments were carried out for catalysts screening, then using the selected catalyst, effects of alcohol and temperature were performed. All experiments were conducted at a stirrer speed of 1000 rpm.

\subsection{Analysis}

Samples taken during the experiments were analyzed using Gas Chromatograph (Agilent 6890A). Hydrogen was used as carrier gas and the column was FFAP. The sample chromatogram was given in Figure 2.

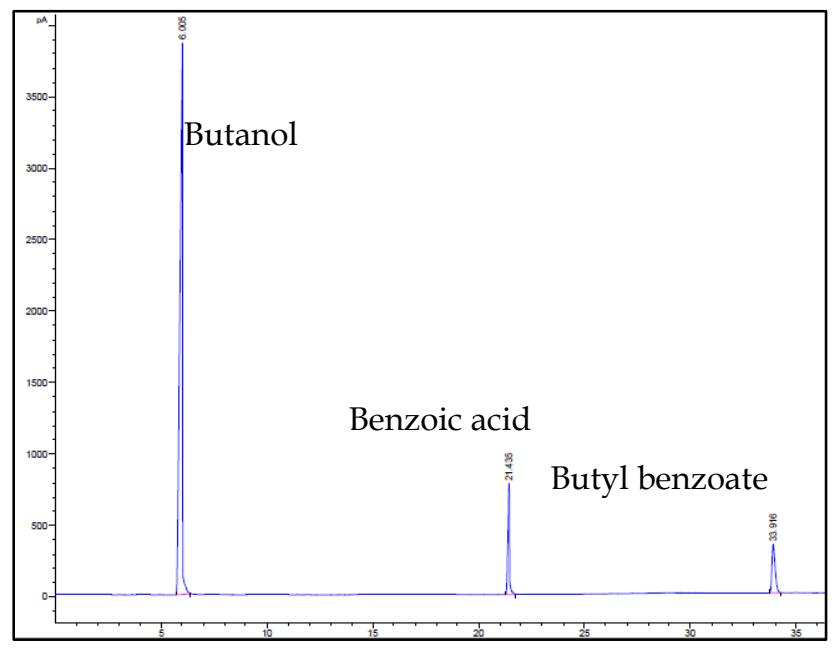

Figure 2. The sample chromatogram for butyl benzoate synthesis

\section{Results and Discussion}

In this study, esterification reactions of benzoic acid with ethanol, butanol and hexanol were investigated. These reactions are equilibrium reactions and slightly exothermic. The equilibrium limits the conversion of benzoic acid, so the catalytic activity of catalyst is very important to accelerate the reaction. The reactions are shown below; ethyl benzoate synthesis:
$\mathrm{C}_{7} \mathrm{H}_{6} \mathrm{O}_{2}+\mathrm{C}_{2} \mathrm{H}_{5} \mathrm{OH}$
$\leftrightarrow \quad \mathrm{C}_{9} \mathrm{H}_{10} \mathrm{O}_{2}+\mathrm{H}_{2} \mathrm{O}$
Benzoic acid + ethanol
$\leftrightarrow \quad$ Ethyl benzoate + water
butyl benzoate synthesis:
$\begin{array}{lll}\mathrm{C}_{7} \mathrm{H}_{6} \mathrm{O}_{2}+\mathrm{C}_{4} \mathrm{H}_{9} \mathrm{OH} & \leftrightarrow & \mathrm{C}_{11} \mathrm{H}_{14} \mathrm{O}_{2}+\mathrm{H}_{2} \mathrm{O} \\ \text { Benzoic acid }+ \text { butanol } & \leftrightarrow & \text { Butyl benzoate }+ \text { water }\end{array}$
hexyl benzoate synthesis:
$\mathrm{C}_{7} \mathrm{H}_{6} \mathrm{O}_{2}+\mathrm{C}_{6} \mathrm{H}_{13} \mathrm{OH} \quad \leftrightarrow \quad \mathrm{C}_{13} \mathrm{H}_{18} \mathrm{O}_{2}+\mathrm{H}_{2} \mathrm{O}$
Benzoic acid + hexanol $\leftrightarrow \quad$ Hexyl benzoate + water

For the esterification of benzoic acid, homogeneous reaction takes place in the presence of ionic liquid and DES, heterogeneous reaction also takes place in presence of Amberlyst 15. By this way, we can compare the catalytic activities of different catalysts as well as the phase of reaction. 


\subsection{Catalyst Screening}

Experiments for catalyst screening were carried at the following conditions; catalyst loading of $10 \mathrm{wt} \%$, stirrer speed of $1000 \mathrm{rpm}$, alcohol/acid mole ratio of 10 and temperatures of 55,65 and $75^{\circ} \mathrm{C}$. Figure 3 represents the conversions of benzoic acid at different temperatures for all catalysts.

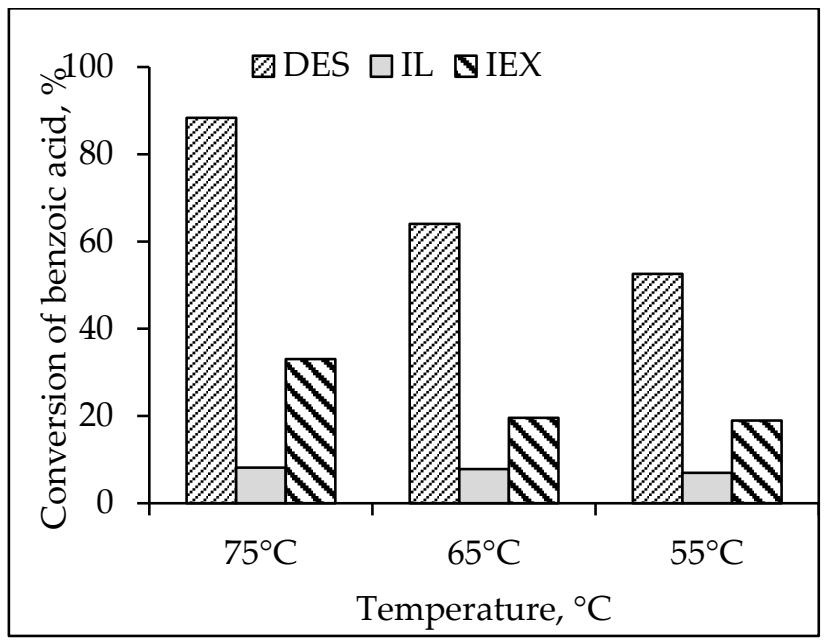

Figure 3. Comparison of catalytic activities of DES, ionic liquid and Amberlyst 15 for the synthesis of ethyl benzoate

As shown from Figure 3, DES composed of ptoluenesulfonic acid and benzyltriethyl ammonium chloride gives the maximum conversion of benzoic acid. At $55^{\circ} \mathrm{C}$, benzoic acid conversion were obtained as 52.7, 18.9 and $69 \%$ for DES, ionic liquid and ion exchange resin, respectively. Both DES and ionic liquid show better catalytic activity than Amberlyst 15 which is commonly used as solid acidic catalyst for esterification reactions. At $65^{\circ} \mathrm{C}, 64,19.6$ and $7.8 \%$ of benzoic acid conversions were achieved for DES, ionic liquid and ion exchange resin, respectively. And also at $75^{\circ} \mathrm{C}$, similar trend was observed, and the maximum conversion was obtained as $88.4 \%$ in run catalyzed by DES. Marziono et.al. [18] studied the esterification of benzoic acid with methanol over $\mathrm{H}_{2} \mathrm{SO}_{4} / \mathrm{SiO}_{2}$ catalyst. Experiments were carried out in presence of toluene and high percentage of methyl benzoate was achieved. In the study of Barbosa [19], benzyl benzoate was synthesized using $\mathrm{H}_{2} \mathrm{SO}_{4}$ and $\mathrm{SiO}_{2}-$ $\mathrm{SO}_{3} \mathrm{H}$ as catalyst. To observe the catalytic acitivity of synthetic silicas, blank experiment was performed, reagents and silica was subjected to microwave radiation, and no products were detected. In presence of catalysts, benzyl benzoate and/or benzyl ether were produced at different extents according to process conditions.

Generally, Amberlyst 15 shows moderate catalytic activity in esterification reactions. In this study, very low conversion values were achieved for the experiments catalyzed by Amberlyst 15, this situation may come from the absence of suitable solvent, higher conversions of benzoic acid may be obtained in presence of suitable solvent. But our aim is to obtain the route without any solvent because of obtaining environmentally friendly operation.

\subsection{Effect of Temperature and Alcohol}

In catalyst screening experiments, DES (p-TSA and BTEAC) was found as the most effective catalyst which gives higher catalytic activity, no side reaction, stability during reaction and no waste.

Effect of temperature on the conversion of benzoic acid was investigated at a catalyst loading of $10 \mathrm{wt} \%$, acid/alcohol molar ratio of 1:10 and stirrer speed of 1000 rpm. Experiments were conducted at three different temperatures; $55^{\circ} \mathrm{C}, 65^{\circ} \mathrm{C}$ and $75^{\circ} \mathrm{C}$. For the synthesis of butyl benzoate, effect of temperature on conversion of benzoic acid was given in Figure 4.

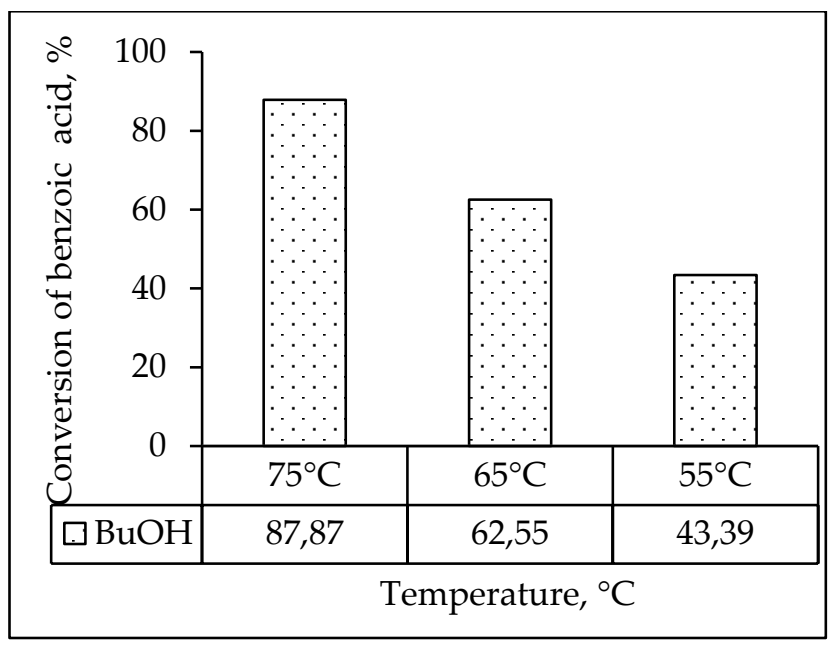

Figure 4. Effect of temperature on conversion of benzoic acid for butyl benzoate synthesis catalyzed by DES composed of $p$-TSA and BTEAC

Increase in temperature from $55^{\circ} \mathrm{C}$ to $65^{\circ} \mathrm{C}$ raises the conversion of benzoic acid from $43.3 \%$ to $62.5 \%$, and further increase in temperature to $75^{\circ} \mathrm{C}$ causes $87.8 \%$ of conversion of benzoic acid. We know that as temperature is increased, number of collisions will increase, so the conversion of limiting reactant will increase by decreasing the activation energy. Wu et. al [20] studied esterification of benzoic acid with ethanol catalyzed by $\mathrm{Ti}_{3} \mathrm{AlC}_{2}$ and $\mathrm{SO}_{4}{ }^{-2} / \mathrm{Ti}_{3} \mathrm{AlC}_{2}$. To determine the effect of temperature, ethyl benzoate synthesis was conducted at different temperatures and $\mathrm{Ti}_{3} \mathrm{AlC}_{2}$ was used as catalyst. 
Conversion of benzoic acid was obtained as $5 \%$ at $95^{\circ} \mathrm{C}$, and 7, 9 and $11 \%$ of conversions of benzoic acid were achieved at temperature of 110,120 and $130^{\circ} \mathrm{C}$, respectively. Then benzoic acid esterification over $\mathrm{SO}_{4}{ }^{-2} /$ $\mathrm{Ti}_{3} \mathrm{AlC}_{2}$ was studied at different temperatures and increase in temperature from $50^{\circ} \mathrm{C}$ to $120^{\circ} \mathrm{C}$ leads to conversion increase from $8 \%$ to $36 \%$ at the end of 12 hours. In our study, increase in temperature lead to higher conversions than solid catalyzed reactions due to absence of mass transfer limitations.

Effect of alcohol on conversion of benzoic acid was studied by the esterification of benzoic acid with ethanol, butanol and hexanol. By this way, effect of chain length of alcohol was observed and also applicability of deep eutectic solvents to an extended range of reactions. Effect of alcohol type on the conversion of benzoic acid was investigated at a catalyst loading of $10 \mathrm{wt} \%$, acid/alcohol molar ratio of $1: 10$ and stirrer speed of $1000 \mathrm{rpm}$ at different temperatures, results were given in Table 1.

Table 1. Effect of alcohol type on conversion of benzoic acid (Catalyst loading of $10 \mathrm{wt} \%$, acid/alcohol molar ratio of 1:10, stirrer speed of $1000 \mathrm{rpm}$, catalyzed by DES)

\begin{tabular}{|c|c|c|}
\hline Temperature $\left({ }^{\circ} \mathbf{C}\right)$ & Alcohol & Conversion (\%) \\
\hline 55 & Ethanol & 52.6 \\
\hline 55 & Butanol & 43.4 \\
\hline 55 & Hexanol & 38.8 \\
\hline 65 & Ethanol & 64.0 \\
\hline 65 & Butanol & 62.5 \\
\hline 65 & Hexanol & 57.8 \\
\hline 75 & Ethanol & 88.3 \\
\hline 75 & Butanol & 87.8 \\
\hline 75 & Hexanol & 67.5 \\
\hline
\end{tabular}

As seen in Table 1, as the length of chain increases, conversion of benzoic acid decreases. At $75^{\circ} \mathrm{C}$, DES provides the high conversions of benzoic acid for longerchained alcohols because of its superior properties such as low viscosity, low $\mathrm{pH}$ value and suitable density values in homogeneous medium. At $75^{\circ} \mathrm{C}$, esterification of benzoic acid gave $88.3,87.8$ and $67.5 \%$ conversion of benzoic acid for ethanol, butanol and hexanol, respectively.

In summary, we have developed an efficient and simple method to produce benzoic acid esters. Among three different catalysts, eutectic solvent showed the highest catalytic activity and stability to accelerate the esterification reactions of benzoic acid. High yields of esters were afforded with same amount of eutectic solvent. After reaction has completed, eutectic mixture can be separated from reaction mixture due to the gravity difference.

\section{Conclusion}

In this study, the catalytic activities of three different catalysts, ion exchange resin, and deep eutectic solvent and ionic liquid were tested for esterification of benzoic acid with ethanol, butanol and hexanol. Generally benzoic acid esters were synthesized using organic solvent. In this study, solvent-free reactions were conducted. High conversions of benzoic acid were achieved in the case of deep eutectic solvent.

As expected, temperature affects the conversion of benzoic acid positively. The maximum conversion was obtained for esterification of benzoic acid with ethanol at a temperature of $75^{\circ} \mathrm{C}$ catalyzed by deep eutectic solvent.

The applicability of deep eutectic solvents in esterification reactions is very important. Besides the catalytic activity, tunability of properties of DES is one of the most important properties. By changing HBA or HBD, different types of DESs can be prepared and also desired properties can be improved.

\section{Acknowledgement}

This study was supported by Ege University (13MÜH66) scientific research project.

\section{References}

1. Othmer, K. Kirk-Othmer Encyclopedia of Chemical Technology, 4th ed.; John Wiley \& Sons: New York, NY, USA, 1994; p. 797.

2. Pipus, G., Plazl, I., Koloini, T., Esterification of benzoic acid in microwave tubular flow reactor, Chemical Engineering Journal, 2000, 76(3), 239-245.

3. Lilja, J., Yu, D., Murzin, Salmi, T., Aumo, J., Mäki-Arvela, P., Sundell, M., Esterification of different acids over heterogeneous and homogeneous catalysts and correlation with the Taft equation, Journal of Molecular Catalysis A: Chemical, 2002, 182, 555-563.

4. Rönnback, R., Salmi, T., Vuori, A., Haario, H., Lehtonen, J., Sundqvist, A., Tirronen, E., Esterification of different acids over heterogeneous and homogeneous catalysts and correlation with the Taft equation, Chemical Engineering Science, 1997, 52, 33693381 .

5. Liu, Y., Lotero, E., Goodwin Jr. J.G., Effect of water on sulfuric acid catalyzed esterification, Journal of Molecular Catalysis A: Chemical, 2006, 245(1-2), 132-140.

6. Buluklu, A.D., Sert, E., Karakus, S., Atalay, F.S., Development of Kinetic Mechanism for the Esterification of Acrylic Acid with Hexanol Catalyzed by Ion-Exchange Resin, International Journal of Chemical Kinetics, 2014, 46, 197-205.

7. Kirumakki, S. R., Nagaraju, N., Chary, K.V.R., Esterification of alcohols with acetic acid over zeolites H 3 , HY and HZSM5, Applied Catalysis A: General, 2006, 299, 185-192. 
8. Sert, E., Atalay, F.S., Esterification of Acrylic Acid with Different Alcohols Catalyzed by Zirconia Supported Tungstophosphoric Acid, Industrial \& Chemistry Research, 2012, 51, 6666-6671.

9. Rajabi, F., Abdollahi, M., Luque, R., Solvent-Free Esterification of Carboxylic Acids Using Supported Iron Oxide Nanoparticles as an Efficient and Recoverable Catalyst, Materials, 2016, 9, 557-563.

10. Gaifutdinova, E. K., Beresnev, V. V., Synthesis of Ethyl Benzoate by Ozonolysis of Styrene in the Presence of Ethanol, Russian Journal of Applied Chemistry, 2002, 75(3), 441-443.

11. Cai, Y.Q., Yu, G.Q., Liu, C. D., Xu, Y.Y., Wang, W., Imidazolium ionic liquid-supported sulfonic acids: Efficient and recyclable catalysts for esterification of benzoic acid, Chinese Chemical Letters, 2012, 23, 1-4.

12. Zhang, Q., Vigier, K. O., Royer, S., Jerome, F., Deep eutectic solvents: syntheses, properties and applications, Chemical Society Reviewes, 2012, 41, 7108-7146.

13. Kareem, M. A., Mjalli, F. S., Hashim, M. A., AlNashef, I. M., Phosphonium-Based Ionic Liquids Analogues and Their Physical Properties, Journal of Chemical Engineering Data, 2010, 55, 46324637.

14. De Santi, V., Cardellini, F., Brinchi, L., Germani, R., Novel Brønsted acidic deep eutectic solvent as reaction media for esterification of carboxylic acid with alcohols, Tetrahedron Letters, 2012, 53, 5151-5155.
15. Taysun, M. B., Sert, E., Atalay, F. S., Physical properties of benzyl tri-methyl ammonium chloride based deep eutectic solvents and employment as catalyst, Journal of Molecular Liquids, 2016, 223, 845-852.

16. Taysun, M. B., Sert, E., Atalay, F. S., Effect of Hydrogen Bond Donor on the Physical Properties of Benzyltriethylammonium Chloride Based Deep Eutectic Solvents and Their Usage in 2Ethyl-Hexyl Acetate Synthesis as a Catalyst, Journal of Chemical \& Engineering Data, 2017, 62(4), 1173-1181.

17. Sert, E., Buluklu, A. D., Karakuş, S., Atalay, F.S., Kinetic study of catalytic esterification of acrylic acid with butanol catalyzed by different ion exchange resins, Chemical Engineering \& Processing, $2013,73,23-28$.

18. Marziano, N.C., Tortato, C. Ronchin, L., Bianchi, C., On the acidity of liquid and solid acid catalysts. Part 3. Esterification of benzoic and mesitoic acids, Catalysis Letters, 2000, 64, 15-22.

19. Barbosa, S. L., Ottone, M., Santos, M. C., Junior, G.C., Lima, C. D.,Glososki, G.C., Lopes, N.P., Klein, S.I., Benzyl benzoate and dibenzyl ether from of benzoic acid and benzyl alcohol under microwave irradiation using a $\mathrm{SiO} 2-\mathrm{SO} 3 \mathrm{H}$ catalyst, Catalysis Communications, 2015, 68, 97-100.

20. Wu, M., Guo, J., Li, Y., Zhang, Y., Esterification of benzoic acid using Ti3AlC2 and SO4 2-/Ti3AlC2 ceramic as acid catalysts, Ceramics International, 2013, 39, 9731-9736. 